ATLAS Internal Note

INDET-NO-120

January 1996

\title{
POSSIBLE LIMITATIONS ON THE OPERATING VOLTAGE OF SILICON DETECTORS IN THE INNER TRACKER
}

\author{
C. Arrighi, J.-C. Clemens, P. Delpierre, E. Grigoriev*, \\ M.-C. Habrard, G. Hallewell, T. Mouthuy, D. Sauvage \\ Centre de Physique des Particules de Marseille \\ 163, Avenue de Luminy, CASE 907, 13288 Marseille, CEDEX 09
}

20 April 1995

Revised 12 December 1995

Presented by E.Grigoriev at the 2nd International Conference on Large Scale Applications and Radiation Hardness of Semiconductor Detectors (June 28-30, 1995, Florence, Italy)

\begin{abstract}
In working out a realistic long-term scenario for the operational conditions for the innermost silicon layers, it is usually assumed that the upper safe margin for the reverse bias voltage is around $200 \mathrm{~V}$. Lifetime of the detectors, and consequently total cost of the tracker are directly related to this value. Using pixel test structures, manufactured by CSEM in the same technology as DELPHI pixel detectors, we performed a study of maximum tolerable bias voltages on nonirradiated detectors, and on detectors which have received different fluences of pions, protons and neutrons. A special test was also done with detectors, continuously irradiated on a testbench with a $\mathrm{Sr}$-source, at a rate about $13 \mathrm{krad} /$ hour.
\end{abstract}

${ }^{*}$ Visitor from ITEP (Moscow); currently Guest Scientist at Max-Planck-Institute for Nuclear Physics, Postfach 103980, D-69029, Heidelberg 


\section{Introduction}

Extensive studies have been made by several groups $[1,2,3]$ in the evaluation of the radiation doses expected in the Si detector layers in particular layouts, for realistic LHC luminosity schedules, and in measuring the effects of such doses on detector performance. Leakage current damage constants and effective impurity concentrations as a function of integrated fluence in beams of different particles $(n, p$ and $\pi)$ have been measured with good precision. Together with annealing and reverse annealing effects, which have been studied at different temperatures, they permitted prediction of the global characteristics (leakage current and full depletion voltage) of the Si detectors throughout the long-term operational scenario at $\mathrm{LHC}[4,5,6,7,8]$.

It has been shown that cooling down the detectors reduces considerably the resultant depletion voltage after 10 years of operation. Nevertheless, some short heatings-up to room temperature for maintenance and repair are unavoidable, and this can considerably increase the final depletion voltage. For example, in a realistic scenario for pixel layers with maintenance at $20^{\circ} \mathrm{C}$ of one month per year at low luminosity and one month per 2 years - at high luminosity, the depletion voltage surpasses $400 \mathrm{~V}$, even for $150 \mu \mathrm{m}$ thick detectors. If this voltage cannot be tolerated by the detectors, they have to be operated in a non-fully depleted mode, leading to partial loss of the signal. Therefore, it is important to understand if the maximum tolerable reverse bias voltage after 10 year of operation will still ensure good detector performance in terms of efficiency and $\mathrm{S} / \mathrm{N}$ ratio.

Though the initial breakdown voltage of nonirradiated detectors is very high, it is determined mainly by the layout and the charge distribution near the surface. The breakdown near the surface occurs at a bias voltage, which is lower than the breakdown voltage in the bulk and can vary significantly under irradiation. Some groups have reported observation of "microdischarges" [9] and excess noise [10] at rather low reverse bias voltages in Si microstrip detectors, which have received relatively small ionization doses.

Therefore we have performed a special study of pre- and post-irradiation breakdown and noise characteristics of the pixel test structures with several guard-ring configurations.

\section{Detector Layout and Irradiation Conditions}

Test structures were fabricated by CSEM (Neuchatel) on $300 \mu \mathrm{m}$ high resistivity n-type substrate on the same wafers as DELPHI pixel detectors [11]. The layout of a test structure is shown schematically in Figure 1. On a surface of about $4.6 \times 5.5 \mathrm{~mm}^{2}$, it contains 16 pixel elements of various sizes, surrounded by individual guard-rings. Two large groups of pixels are in addition surrounded by external guard-rings, which are close to the scribe lines; metallized surfaces over- 
lap implantation zones by $10 \mu \mathrm{m}$. The pixels of "DELPHI-type" $330 \times 330 \mu \mathrm{m}^{2}$ were used for breakdown and noise studies. Two types of structures were available - 300 and $165 \mu \mathrm{m}$ thick, but only the thick ones have been irradiated.

In the period June-July 1994 irradiations of test-structures were performed in the following beams:

- neutrons with average kinetic energy of $6.2 \mathrm{MeV}$ at SARA(Grenoble) up to fluences of $2.7 * 10^{14} \mathrm{~cm}^{-2}$;

- protons with kinetic energy of $300 \mathrm{MeV}$ at SATURNE(Saclay) up to fluences of $2.6 * 10^{14} \mathrm{~cm}^{-2}$;

- positively charged pions with kinetic energy of $238 \mathrm{MeV}$ at PSI (Villigen) up to fluences of $1.3 * 10^{14} \mathrm{~cm}^{-2}$.

Irradiations by pions were done at room temperature, by neutrons - at $+5^{\circ} \mathrm{C}$, by protons - at both temperatures simulteneously. After irradiations all samples were kept in a refrigerator at a temperature of $-20^{\circ} \mathrm{C}$. No bias was applied at either stage. All measurements were done about 180 days after the irradiations.

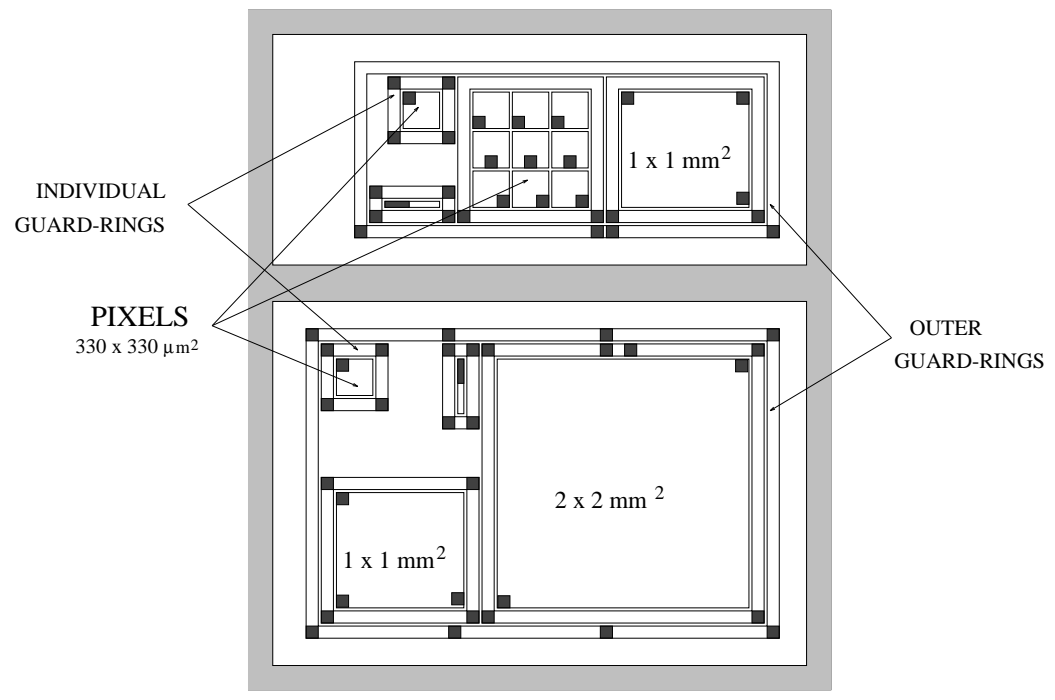

Figure 1: Layout of a pixel test structure manufactured by CSEM.

The I-V measurements were performed at room temperature on a probe station with temperature monitoring and dry-air ventilation (to suppress stray currents). A KEITHLEY-237 served as a voltage supply and current-measuring device with a resolution of $0.1 p A$. Current was measured on a single element at a time, several neighbouring pixels and guard-rings being kept at the same potential. 


\section{Breakdown Voltage from the I-V Curves}

I-V curves for single pixels on nonirradiated detectors were measured in two different guard-ring connection configurations: a) individual guard-ring of the pixel and the outer guard-ring are at the same potential as the pixel; b) only individual guard-ring is connected, the outer guard-ring being floating. The breakdown in configuration a) occurs at a voltage around $550 \mathrm{~V}$, in configuration b) - around $820 \mathrm{~V}$, with a spread of $\sim \pm 10 \mathrm{~V}$ for different detectors. The shapes of the curves and the values of breakdown voltages are about the same for 165 and $330 \mu \mathrm{m}$ thick detectors: this indicates that the breakdown is caused by surface effects. For comparative studies of different detectors the breakdown voltage was determined as the one at which current grows by more than $20 \%$ with a voltage increase of $20 \mathrm{~V}$.

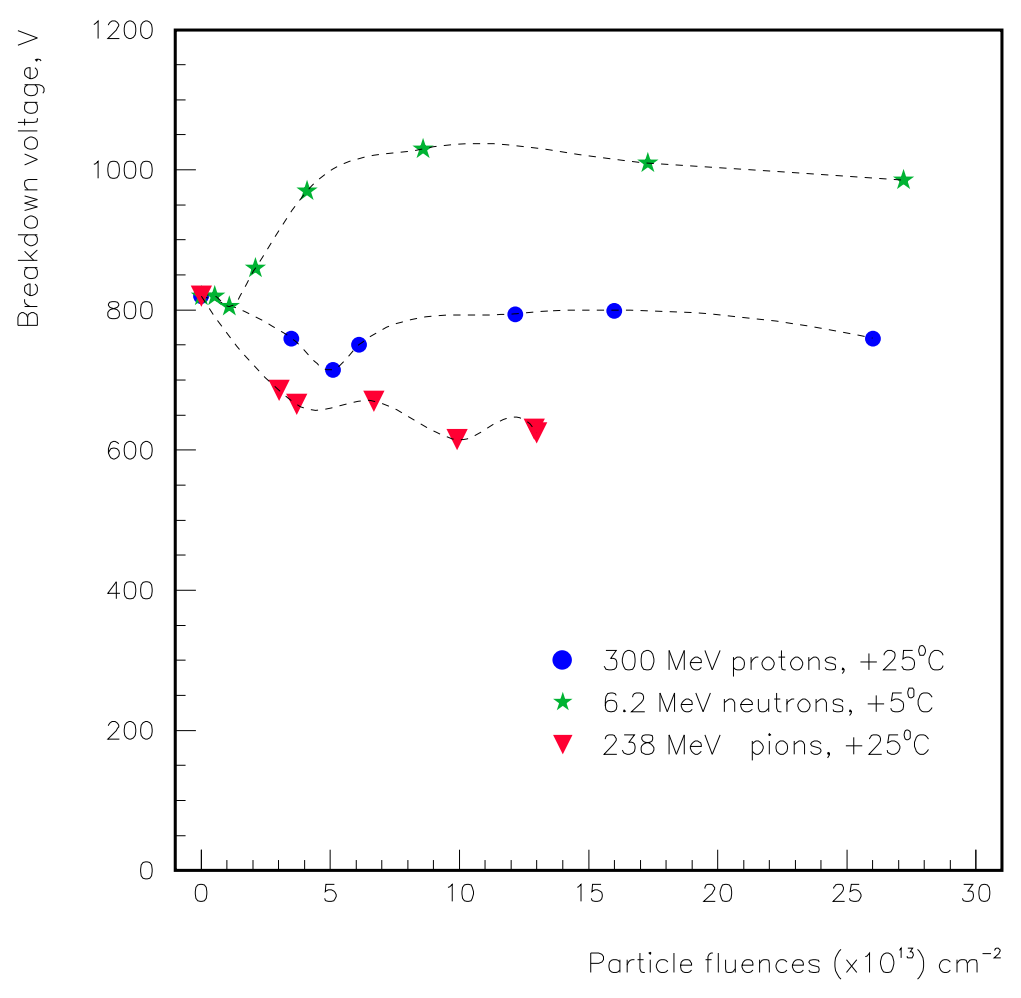

Figure 2: Breakdown voltage for irradiated detectors.

The same kind of measurements were performed with irradiated detectors. For this purpose they were taken out of the refrigerator and kept at room temperature for a period necessary for the measurements. The summary of all 
measurements for the detectors irradiated with different particles is represented by curves in Figure 2 .

With the common starting point of $820 \mathrm{~V}$ (external guard-ring being floating in all cases), there are quite different tendencies observed in different groups: while neutrons somehow "harden" breakdown properties of the detectors, irradiation by pions noticably degrades them; the effects of protons being somewhat intermediate. Such kind of behaviour well agrees with the assumed surface nature of the breakdown effects. The influence of temperature during irradiation on the breakdown voltage was checked on the proton-irradiated samples. Figure 3 demonstrates that breakdown voltage for the detectors kept in a cool-box $\left(+5^{\circ} \mathrm{C}\right)$ during irradiation is systematically slightly lower than that for the detectors irradiated at room temperature.

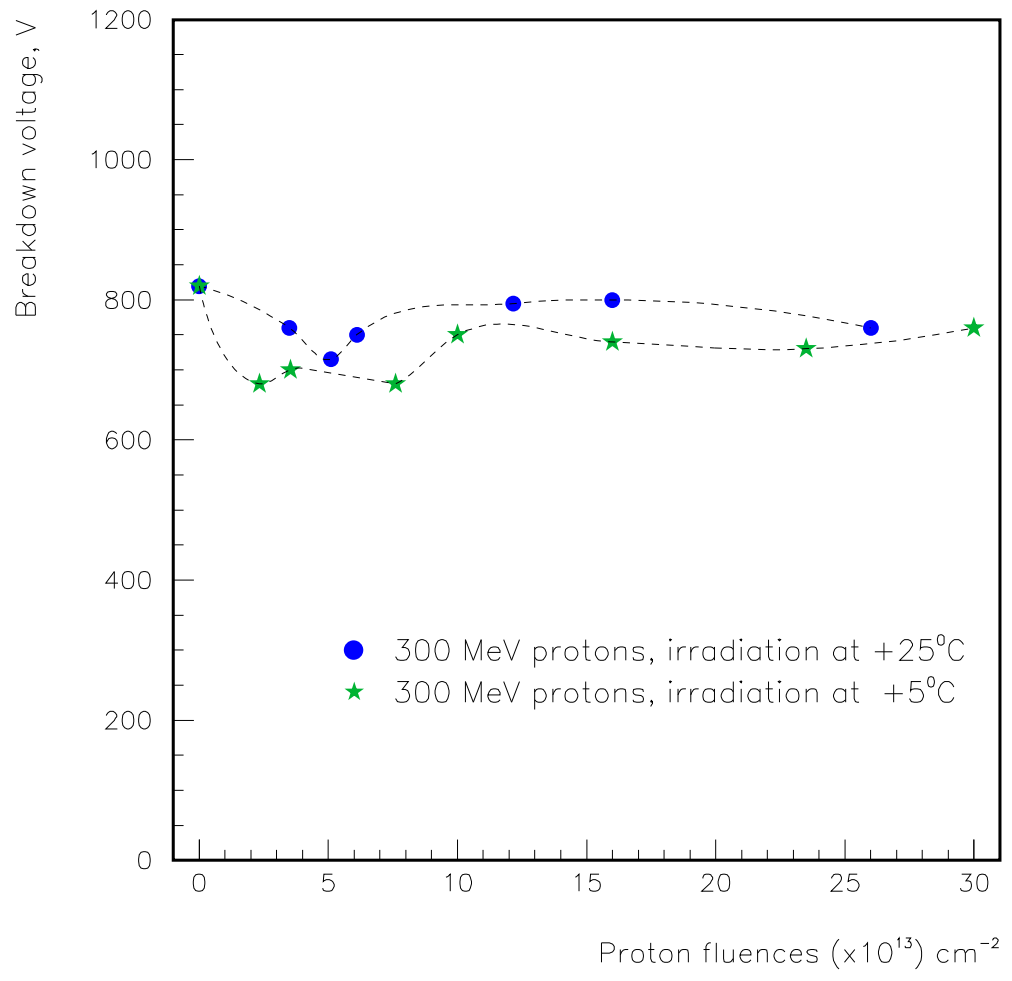

Figure 3: Breakdown voltage for detectors irradiated at different temperatures

Though irradiation by ionizing particles reduces the breakdown voltage of the detector, as determined from the I-V characteristic, it still remains well above $500 \mathrm{~V}$, suggesting a safe margin for increased working voltage of detectors over the LHC operational period. Most likely the upper margin for the bias will 
be determined by excess noise, appearing at lower bias voltages.

\section{Measurement of Noise on Irradiated Detec- tors}

Pulses produced by electrons from a Sr-source in nonirradiated detectors, and several detectors irradiated by neutrons and pions, were measured with a chain of amplifiers having total equivalent noise of about 800 electrons. Therefore, only noise well above this level was considered significant, and was studied as a function of irradiation dose and reverse bias voltage.

The main components of the system were: LeCroy charge - sensitive amplifier HQV-820M; wide-band amplifier PHILLIPS SCIENTIFIC 6954 and the timing filter amplifier ORTEC-474 (shaping time set to $20 \mathrm{nsec}$ ). Pulseheight spectra were measured by LeCroy ADC 2259B; pulses and noise were also observed directly on a fast digital scope. The detector under study was put between the Sr-source and a scintillation counter which provided a trigger in coincidence with the signal, produced by pixel itself over a certain threshold (slightly above the system noise). Noise studies were performed in self-triggered mode.

The spectrum of noise in the system and a typical MIP-spectrum from a single pixel (see Fig.1), which were measured separately, are superimposed in Figure 4 . The whole surface of the detector (all diodes and guard-rings, except for the outer one) had the same potential during the measurements. The lower wing of the Landau curve is destorted by pulses produced by particles crossing between the pixel and the guard-ring, which should not be taken for the noise. The lower edge of the MIP-spectrum indicates the discriminator threshold, which was set at a level of about 3 times the total noise of the electronic system for detector noise studies.

The detector noise appears at relatively high bias and is visible in Figure 5 as an excess on the left side of the curve, going down exponentially towards higher amplitudes and overlapping to a large extent with the MIP-spectrum. Curves presented in Figure 4 and Figure 5 were measured on a single pixel of a non-irradiated detector and correspond to bias voltages of 770V (Fig.4) and $860 \mathrm{~V}$ (Fig.5). In this case the noise threshold voltage well matches with the breakdown $(820 \mathrm{~V})$, which is not the case for detectors, irradiated with ionizing particles. The noise rate and spectrum are not correlated with the presence of the Sr-source (in all cases).

In the detectors irradiated with ionizing particles, noise appeares at lower voltages, depending on the dose; its ampiltude and rate grow slowly with increase of the bias voltage until a real breakdown occurs. It is clearly visible on the scope in random trigger mode as fast sporadic pulses, which appear at a certain threshold voltage and have sometimes an amplitude comparable with 


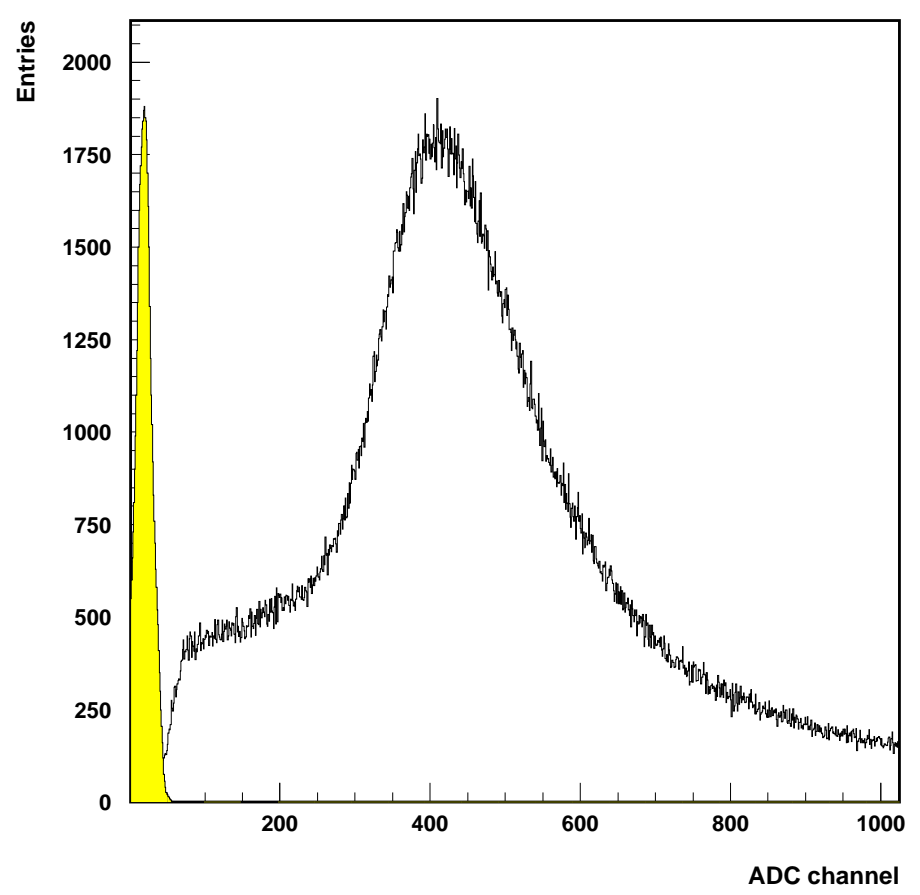

Figure 4: Spectrum from a Sr-source at $770 \mathrm{~V}$ on nonirradiated detector

that from a MIP, threshold being not correlated with the leakage current. A spectrum corresponding to the same detector as in Figures 4-5, but measured at $\mathrm{V}=210 \mathrm{~V}$ immediately after irradiation by a Sr-source with a dose of $750 \mathrm{Krad}$, looks very similar to one shown in Fig.5.

The threshold voltage, at which the noise appears, can be well determined both from the rise in the RMS-amplitude of the pulses observed on the scope in random trigger and from the shape of a MIP-spectrum. This voltage was measured for several detectors at room and low $\left(-5^{\circ} \mathrm{C}\right)$ temperatures in different guard-ring configurations. It was found that the noise threshold voltage is very high (around 1000V, like the breakdown voltage) for neutron-irradiated saples and about $390 \mathrm{~V}$ - for pion-irradiated ones, independent of fluence. The last value is twice higher than that observed on the Sr-irradiated test-structures, which received about 10 times less dose, but were measured just after irradiation.

Results, obtained from different test-structures before and after their irradiation by a Sr-source up to $1 \mathrm{Mrad}$; by pions up to $6.7 * 10^{13}$ and $1.3 * 10^{14} \mathrm{~cm}^{-2}$ and by neutron fluences of $0.86 * 10^{14}$ and $2.72 * 10^{14} \mathrm{~cm}^{-2}$, can be summarized as follows:

- the noise threshold voltage is not correlated with detector leakage current; 


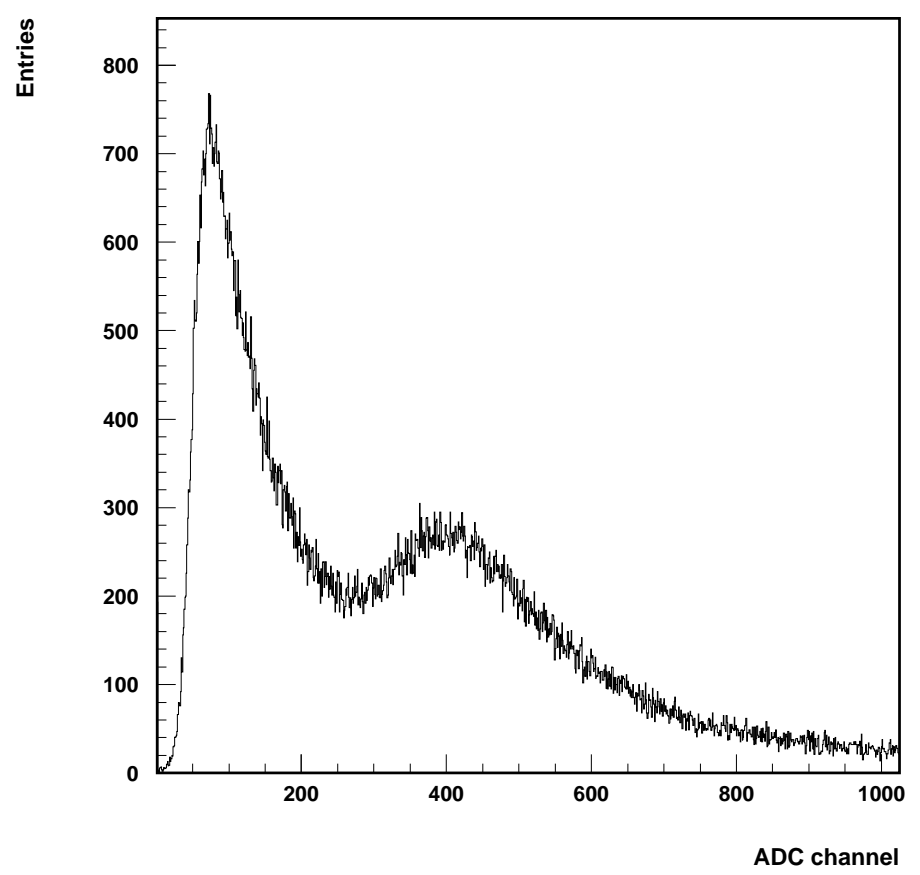

Figure 5: Spectrum from a Sr-source at $860 \mathrm{~V}$ on nonirradiated detector

- for nonirradiated and neutron-irradiated detectors the noise threshold well coincides with the conventional breakdown visible on the I-V curves;

- the noise occurs at higher voltages if the external guard-ring is floating;

- cooling down the detector from room temperature to $-5^{\circ} \mathrm{C}$ slightly shifts the threshold voltage down (typically by $10 \mathrm{~V}$ );

- there are indications on saturation and self-annealing of the noise properties of the detectors.

These observations suggest that the noise is due to surface effects and therefore is determined mainly by ionizing damage. In order to study the effect more accurately as a function of ionizing doses, bench irradiations of two detectors with a Sr-source and their periodical characterization were performed in the lab. Initially the noise appeared at $500 \mathrm{~V}$ in one detector and at $700 \mathrm{~V}$ - in the other one (external guard-ring was floating in both cases). This difference can be explained by some small initial doses already recieved by these detectors in earlier tests. This threshold was periodically remeasured in the course of irradiation at a rate of $12.7 \mathrm{krad} /$ hour up to the maximal dose of 1 Mrad. All measured points are put on the plot shown in Figure 6. Local bumps reflect different short tests during irradiation (switching off the bias or changing polarity), but the general 
trend of exponential fall is obvious; this could explain the fact that the excess noise observed by CDF [10] appeared at relatively low doses. At doses of about $1 \mathrm{Mrad}$, the noise appears at a voltage of about $200 \mathrm{~V}$. Since it was not observed below this voltage in the detectors irradiated by pions and protons up to much higher doses (10 Mrad), which were measured 1-2 months after irradiation, it is possible that due to oxide charge saturation (which is known to occur around $3 * 10^{12} \mathrm{~cm}^{-2}$ ) and annealing effects, degradation could stop at such values.

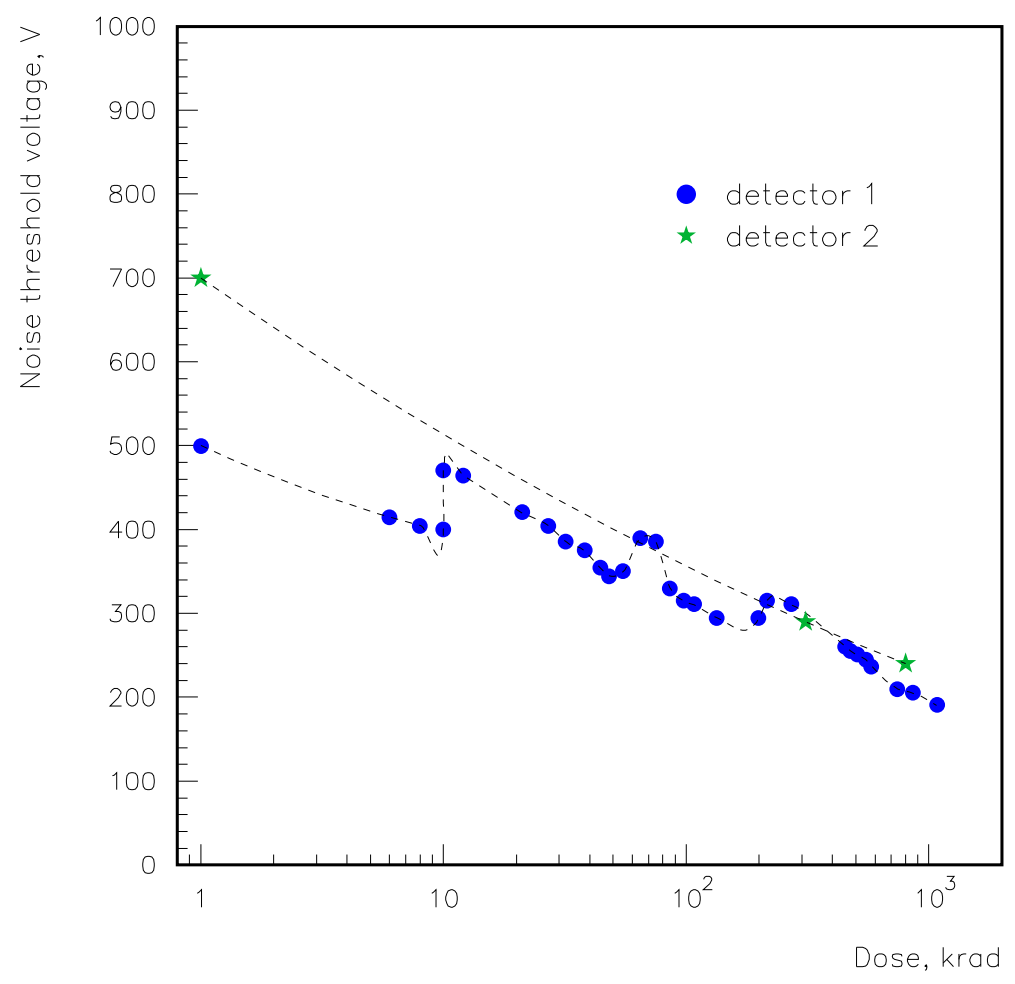

Figure 6: Noise threshold voltage as a function of dose

It was noticed that even a short interruption of the bias or polarity inversion brings the noise threshold up by $50-60 \mathrm{~V}$, but it cures the problem only for a short time: degradation after that goes faster until the points fall on the same curve. Neither cooling the detector down to $-5^{\circ} \mathrm{C}$, nor heating it up to $100^{\circ} \mathrm{C}$ (for one hour), changed the result. 


\section{Conclusions}

A series of breakdown and noise measurements was made on similar (produced together on the same wafer) pixel test-structures before and after irradiation in neutron, proton and pion beams. It was observed that the breakdown (i.e. steep increase of current-voltage characteristic) occurs at relatively high reverse bias voltages (about $800 \mathrm{~V}$ for nonirradiated detectors and about $600 \mathrm{~V}$ in the worst case of pion-irradiated ones). From this point of view a safety margin defined for operation at LHC could be rather high.

Nevertheless, there are other effects, most likely of surface nature, which impose more severe upper limits on the bias voltage. Excess noise, which has been also observed by other groups, appeares at voltages much lower than the breakdown voltage. Detailed studies of this noise in different conditions on the detectors irradiated in different beams to various fluences and by Sr-source point to its surface origin. Therefore, even relatively small doses of ionization can considerably degrade the performance of detectors, fabricated in conventional technology. Standard high-resistivity n-type detectors with usual guard-ring configuration can be operated at $200 \mathrm{~V}$ maximum after having received ionizing dose of 1 Mrad. Hopefully due to saturation of the surface charge and selfannealing, further degradation does not occur far beyond such doses. The fact that the breakdown does not depend on the detector thickness also favours its reduction, but one should also take into account the detector noise in calculating $\mathrm{S} / \mathrm{N}$ ratio. To extend the limits for operating voltage and therefore prolong lifetime of pixel detectors in heavy radiation environment, special care should be taken in design of layout and guarding structure (see for example $[12,13]$ ).

\section{References}

[1] T.Mouthuy "Radiation Doses Expected at LHC", ATLAS INDET-NO-028, October 6, 1993

[2] G.Gorfine and G.Taylor "Particle Fluxes and Damage to Silicon in the ATLAS Inner Detector", ATLAS INDET-NO-030, November 1993

[3] M.Huhtinen, P.A.Aarnio "Neutron and Photon Fluxes and Shielding Alternatives for the CMS Detector at LHC", CMS TN/94-241 (1994)

[4] S.J.Bates, D.J.Munday, M.A.Parker et al., RD2-collaboration, CERN/ECP 94-2, March 1994; presented at 5th Topical Seminar on Experimental Apparatus for Particle and Astrophysics, San Miniato, Italy, April 1993

[5] SCT group, ATLAS Internal Note INDET-NO-085 (1994)

[6] RD20 Status Report, CERN/DRDC/94-39, 1994 
[7] A.Chilingarov and S.Roe "Radiation Damage Projections: Current Status", ATLAS INDET-NO-031, November 1993

[8] E.Fretwurst, H.Feick, M.Glaser et al. "Reverse Annealing of the Effective Impurity Concentration and Long-Term Operational Scenario for Silicon Detectors in Future Collider Experiments", RD2-collaboration; CERN/ECP 93-17; presented at the International Symposium on Development and Application of Semiconductor Tracking Detectors, Hiroshima, Japan, May 1993

[9] T.Ohsugi, Y.Iwata, H.Ohyama et al. "Micro-Discharges of AC-coupled Silicon Strip Sensors", preprint KEK 93-129 (October 1993); Presented at the International Symposium on Development and Application of Semiconductor Tracking Detectors, Hiroshima, Japan, May 1993

[10] N.Bacchetta and D.Bisello, communications at CMS and RD20 meetings at CERN on radiation hardness, January-February 1995

[11] DELPHI collaboration, "Pixel detector for LEP200", Proceedings of Wire Chamber Conference, Wien, February 1995

[12] L.Evensen, A.Hanneborg, B.Sundby Avset and M.Nese, "Guard Ring Design for High Voltage Operation of Silicon Detectors", Proceedings of the Sixth Symposium on Semiconductor Detectors, Milano, February 1992

[13] A.Bischoff, N.Findeis, D.Hauff et al. "Breakdown Protection and LongTerm Stabilisation for Si-Detectors", NIM, A326 (1993) 27-37 